\title{
MASK IMAGE GENERATION FOR SEGMENTING RETINAL FUNDUS IMAGE FEATURES INTO ISNT QUADRANTS USING ARRAY CENTROID METHOD
}

\author{
Darsana $S^{1}$, Rahul M Nair ${ }^{2}$ \\ ${ }^{I}$ MTech Student, Department of ECE, Nehru College of Engineering and Research Centre Thrissur, Kerala, India \\ ${ }^{2}$ Assistant Professor, Department of ECE, Nehru College of Engineering and Research Centre Thrissur, Kerala, India
}

\begin{abstract}
Retinal fundus image processing paves the way for diagnosis of many eye disorders. The fundus image features such as optic disc, optic cup, optic rim and blood vessels help to analyse the pathological condition of eye. The analysis involves calculation of ocular parameters such as Rim to Disc Ratio (RDR) and Inferior Superior Nasal Temporal (ISNT) Ratio and verification of ISNT Rule. For this analysis the fundus image features have to be segmented into ISNT quadrants. This paper presents a novel approach towards mask image generation for segmenting fundus image feature into ISNT quadrants using array centroid method. The existing method uses a predefined mask image which is not accurate and the proposed array centroid method overcomes the limitations of existing method. The method involves centroid calculation, array initialization; mask image generation and, mask- feature image multiplication. The proposed method is applied to the fundus images collected from a reputed eye hospital.
\end{abstract}

Keywords: Fundus Image Features, Array Centroid Method, Centroid Calculation, Mask Image Generation

\section{INTRODUCTION}

Image processing is a vital field of research and has been established as a core field of innovation in modern health care. Among the processing techniques medical image segmentation plays an important role in biomedical applications. The retinal fundus image processing helps to identify eye disorders such as glaucoma, diabetc retinopathy, hypertension, macular edema, arteriosclerosis etc. Fundus image processing involves fundus image feature extraction and its analysis. Inorder to analyse the fundus features some ocular parameter have to be calculated. For this the features have to be segmented into the ISNT quadrants. In the existing methods predefined mask images of size same as that of the image are used to segment the features into four quadrants. In such cases centroid of the feature is not considered and thus results in inaccurate segmentation of the features. This paper proposes a novel approach towards mask generation for ISNT quadrants using array centoid method. This method involves optic disc centroid calculation, array initialization, mask generation and mask-feature image multiplication.

The paper is organized as follows. The section 2 gives theoretical background which includes basics of fundus eye image, fundus image features and ISNT quadrants. Section 3 describes the proposed array centroid method. Section 4 shows the experimental results. Conclusion and future scope are presented in section 5 .

\section{THEORETICAL BACKGROUND}

The basics of fundus image, fundus image features and ISNT quadrants are explained below.

\subsection{Fundus Image}

The fundus of the eye is the interior surface of the eye, opposite to the lens, and includes the retina, optic disc, macula, fovea, and posterior pole. Fundus photography (also called fundography) is the creation of a photograph of the fundus. Modern fundus photographs generally recreate considerably larger areas of the fundus than what can be seen with handheld ophthalmoscopes. Fundus photography is performed by a fundus camera, which basically consists of a specialized low power microscope with an attached camera. Fundus image for a left eye is shown in Fig 1.

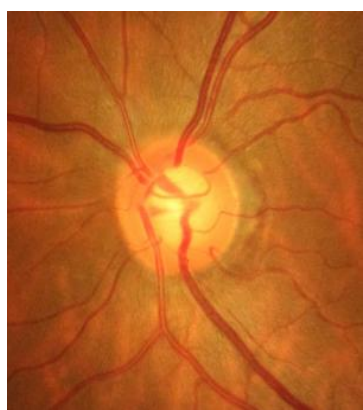

Fig -1: Fundus eye image 


\subsection{Fundus Image Features}

The important fundus image features are optic disc, optic cup, optic rim and blood vessels as shown in Fig2. The optic disc or optic nerve head is the location where ganglion cell axons exit the eye to form the optic nerve. The optic disc is also the entry point for the major blood vessels that supply the retina. There is a central depression, of variable size, called the optic cup.

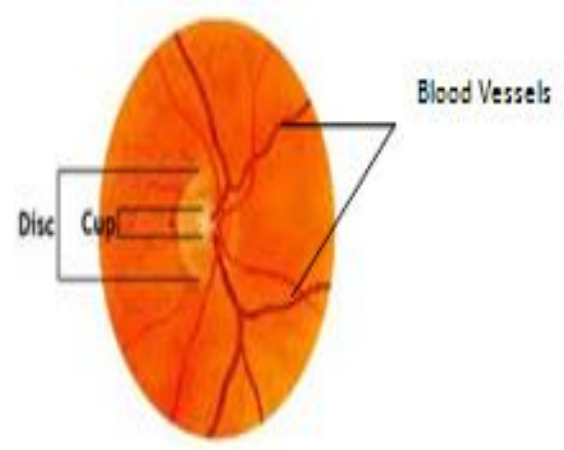

Fig -2: Fundus eye image features

Optic rim is the region between the optic disc and optic cup. The pattern of rim width in a healthy optic nerve is thickest inferiorly, followed by superiorly, nasally and the temporal rim is the thinnest.

\subsection{Extracted Fundus Eye Features}

The fundus eye image features can be extracted from the fundus color image using image processing techniques such as color space analysis, morphological processing, filtering and thresholding. The binary images of fundus features for the image shown in Fig 1 is as shown in Fig 3

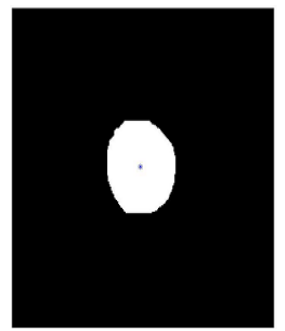

(a)

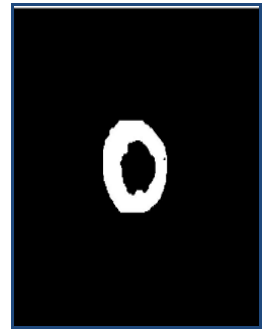

(b)

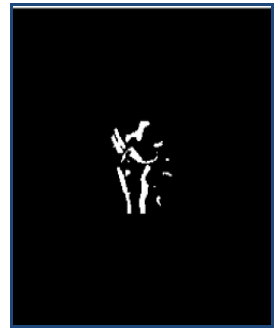

(c)
Fig -3: Binary images of fundus image features (a) Optic disc (b) Optic rim (c) Blood vessels in disc

\subsection{Fundus Image}

The Inferior Superior Nasal and Temporal quadrants for retinal image are as shown in Fig 4. Inferior quadrant is the lowermost quadrant and superior quadrant is the uppermost quadrant. The nasal quadrant is the right quadrant in right eye and left quadrant in left eye. The temporal quadrant is the left quadrant in right eye and right quadrant in left eye.

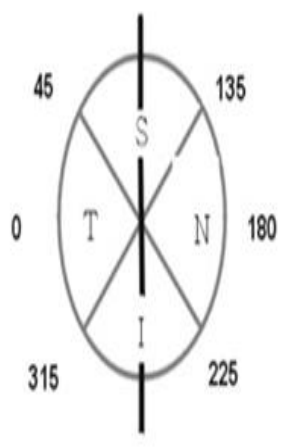

RIGHT EYE

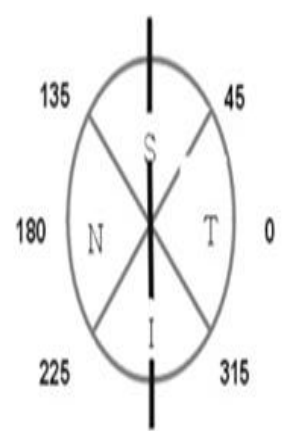

LEFT EYE
Fig -4: ISNT quadrants

The relevance of these quadrants in fundus image analysis is that the segmented fundus eye features in the ISNT quadrants can be used for the calculation of ISNT ratio, Rim to Disc Ratio (RDR) and ISNT rule verification which further helps in the diagnosis of many eye disorders. The cardinal clinical sign of glaucomatous optic neuropathy is thinning of the neuroretinal rim, with the regional preference for the superior and inferior poles of the optic disc. RDR is calculated for the inferior and superior quadrant as the ratio of rim area to disc area. ISNT ratio is calculated using the ratio of area of blood vessels in inferior superior to nasal temporal side of optic disc. There may be a shift in blood vessels to nasal side in glaucoma patients and thus the ISNT ratio will be less compared to normal person's image. The ISNT rule states that in normal eyes the thickness of the neuroretinal rim along the cardinal meridians of the optic disc ,that is the rim width, decreases in the order inferior (I), superior $(\mathrm{S})$, nasal $(\mathrm{N})$, temporal $(\mathrm{T})$, and that the neuroretinal rim in glaucomatous optic disc violates this quantitative relationship. The four variants of $\mathrm{ISNT}$ rule are $(\mathrm{I}>\mathrm{S}, \mathrm{I}>\mathrm{S}>\mathrm{T}, \mathrm{I}>\mathrm{T}$, and combined $\mathrm{I}>\mathrm{T}$ and $S>T$ ).

\section{METHODOLOGY}

The flow chart for the array centroid method is shown in Fig 5. The method involves centroid calculation, array initialization, mask image generation and mask- feature image multiplication. 


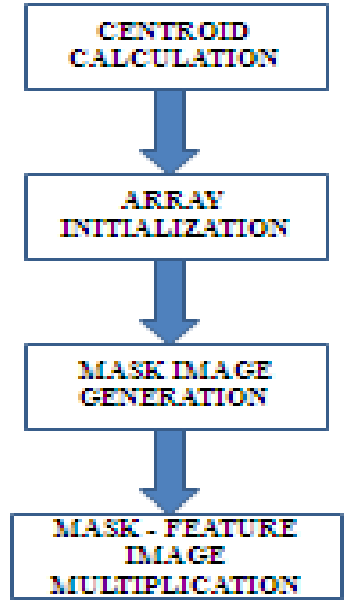

Fig -5: Flow chart of Array Centroid Method

\subsection{Centroid Calculation}

The first step in array centroid method is the calculation of centroid of the optic disc. The centrod value of optic disc is calculated and the centroid is located in the image as shown in Fig 6. The centroid values are denoted as $(\mathrm{C} 1, \mathrm{C} 2)$.

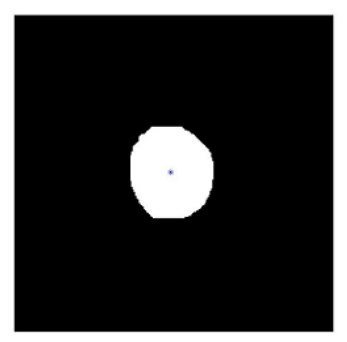

Fig -6: Centroid of optic disc

\subsection{Array Initialization}

The Array consists of rows and columns and the size of the array is defined by this rows and columns. The array initialization involves defining an array of size same as that of the image to be segmented and then initializing all its values with zero as shown in Fig 7. The image size is defined by its pixels and each pixels are denoted as $(\mathrm{i}, \mathrm{j})$. The number of rows and columns in array are denoted as $\mathrm{R}$ and $\mathrm{C}$ respectively.

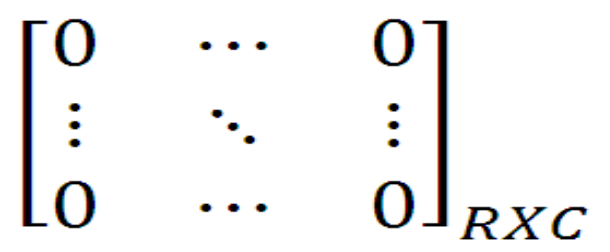

Fig -7: Initialized array

\subsection{Mask Image Generation}

The mask image for each quadrant is generated exploiting the characteristics of array and the centroid value. The mask image act as an image filter Thus the mask image should be such that the desired quadrant should be white and all other quadrants should be black. The mask generation for the four different quadrants are explained below.

Inferior quadrant is the lowermost quadrant. The mask for this quadrant should be such that inferior quadrant is white or binary 1 and all other quadrants are black or binary 0.The logic used to generate inferior quadrant mask is given below.

For $\mathrm{i}$ varies from $\mathrm{C} 2$ to $\mathrm{R}$ and $\mathrm{j}$ varies from 1 to $\mathrm{C}$, set the pixels 0 for following condition and all other pixels as 1

\begin{tabular}{|l|l|}
\hline $\mathrm{C} 1=\mathrm{C} 2$ & $\mathrm{i}+\mathrm{j}<\mathrm{C} 1+\mathrm{C} 2$ or $\mathrm{i}<\mathrm{j}$ \\
\hline $\mathrm{C} 1>\mathrm{C} 2$ & $\mathrm{i}+\mathrm{j}<\mathrm{C} 1+\mathrm{C} 2$ or $\mathrm{i}<\mathrm{j}$ and $\mathrm{j}-1>\mathrm{C} 1-\mathrm{C} 2$ \\
\hline $\mathrm{C} 1<\mathrm{C} 2$ & $\mathrm{i}+\mathrm{j}<\mathrm{C} 1+\mathrm{C} 2$ or $\mathrm{i}<\mathrm{j}$ or $\mathrm{i}=\mathrm{j}$ or $\mathrm{i}-\mathrm{j}<\mathrm{C} 2-\mathrm{C} 1$ \\
\hline
\end{tabular}

Superior quadrant is the uppermost quadrant and the mask image should be such that superior quadrant is white or binary 1 and all other quadrants are black or binary 0 . The logic used to generate superior quadrant mask is given below.

For $\mathrm{i}$ varies from 1 to $\mathrm{C} 2 \mathrm{j}$ varies from 1 to $\mathrm{C}$, set the pixels 0 for following condition and all other pixels as 1

\begin{tabular}{|l|l|}
\hline $\mathrm{C} 1=\mathrm{C} 2$ & $\mathrm{i}+\mathrm{j}>\mathrm{C} 1+\mathrm{C} 2$ or $\mathrm{i}>\mathrm{j}$ \\
\hline $\mathrm{C} 1>\mathrm{C} 2$ & $\mathrm{i}+\mathrm{j}>\mathrm{C} 1+\mathrm{C} 2$ or $\mathrm{i}>\mathrm{j}$ or $\mathrm{i}=\mathrm{j}$ or $\mathrm{j}-1<\mathrm{C} 1-\mathrm{C} 2$ \\
\hline $\mathrm{C} 1<\mathrm{C} 2$ & $\mathrm{i}+\mathrm{j}>\mathrm{C} 1+\mathrm{C} 2$ or $\mathrm{i}>\mathrm{j}$ and $\mathrm{i}-\mathrm{j}>\mathrm{C} 2-\mathrm{C} 1$ \\
\hline
\end{tabular}

The leftmost quadrant is the nasal quadrant for left eye and temporal quadrant for the right eye. The mask for left quadrant has white pixels on left quadrant and black pixels on other quadrants. The logic used for the mask generation of left quadrant is shown below.

\begin{tabular}{|l|l|}
\hline $\begin{array}{l}\text { For } i \text { varies from } 1 \text { to } \mathrm{R} j \text { varies from } 1 \text { to } \mathrm{C} 1 \text {,set the } \\
\text { pixels } 0 \text { for following condition and all other pixels as } 1\end{array}$ \\
\hline $\mathrm{C} 1=\mathrm{C} 2$ & $\mathrm{i}+\mathrm{j}>\mathrm{C} 1+\mathrm{C} 2$ or $\mathrm{i}<\mathrm{j}$ \\
\hline $\mathrm{C} 1>\mathrm{C} 2$ & $\mathrm{i}+\mathrm{j}>\mathrm{C} 1+\mathrm{C} 2$ or $\mathrm{i}<\mathrm{j}$ and $\mathrm{j}-1>\mathrm{C} 1-\mathrm{C} 2$ \\
\hline $\mathrm{C} 1<\mathrm{C} 2$ & $\mathrm{i}+\mathrm{j}>\mathrm{C} 1+\mathrm{C} 2$ or $\mathrm{i}<\mathrm{j}$ or $\mathrm{i}=\mathrm{j}$ or $\mathrm{i}-\mathrm{j}<\mathrm{C} 2-\mathrm{C} 1$ \\
\hline
\end{tabular}

The rightmost quadrant is the temporal quadrant for left eye and nasal quadrant for the right eye. The mask for right quadrant has white pixels on right quadrant and black pixels on other quadrants. The logic used for the mask generation of right quadrant is shown below. 
For $\mathrm{i}$ varies from 1toR $\mathrm{j}$ varies from $\mathrm{C} 1$ to $\mathrm{C}$, set the pixels 0 for following condition and all other pixels as 1 $\mathrm{C} 1=\mathrm{C} 2 \quad \mathrm{i}+\mathrm{j}<\mathrm{C} 1+\mathrm{C} 2$ or $\mathrm{i}>\mathrm{j}$

$\mathrm{C} 1>\mathrm{C} 2 \quad \mathrm{i}+\mathrm{j}<\mathrm{C} 1+\mathrm{C} 2$ or $\mathrm{i}>\mathrm{j}$ or $\mathrm{i}=\mathrm{j}$ or $\mathrm{j}-1<\mathrm{C} 1-\mathrm{C} 2$

$\mathrm{C} 1<\mathrm{C} 2 \quad \mathrm{i}+\mathrm{j}<\mathrm{C} 1+\mathrm{C} 2$ or $\mathrm{i}>\mathrm{j}$ and $\mathrm{i}-\mathrm{j}>\mathrm{C} 2-\mathrm{C} 1$

\subsection{Mask Feature Image Multiplication}

Inorder to segment the fundus features into the ISNT quadrants the feature image have to be multiplied with the respective quadrant mask image. Thus the optic disc, optic rim and blood vessel in each of the four quadrants are obtained.

\section{EXPERIMENTAL RESULTS}

The proposed method is applied to the image database collected from hospital. The different fundus image features are segmented into the four quadrants using array centroid method.

The mask generated for the ISNT quadrants are shown in Fig 8

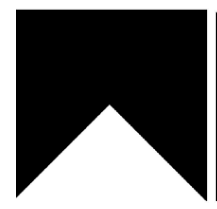

(a)

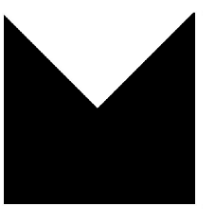

(b)

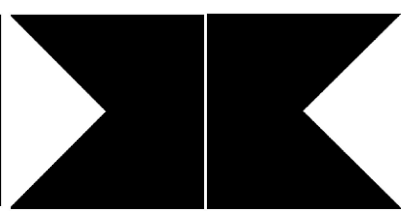

(c) (d)
Fig -8: Mask generated for (a) inferior quadrant (b) superior quadrant (c) left quadrant (d) right quadrant

The retinal fundus image features such as optic disc, optic rim and blood vessels in disc region are segmented into the ISNT quadrants using the mask generated and the segmented results are shown in Fig 9 .

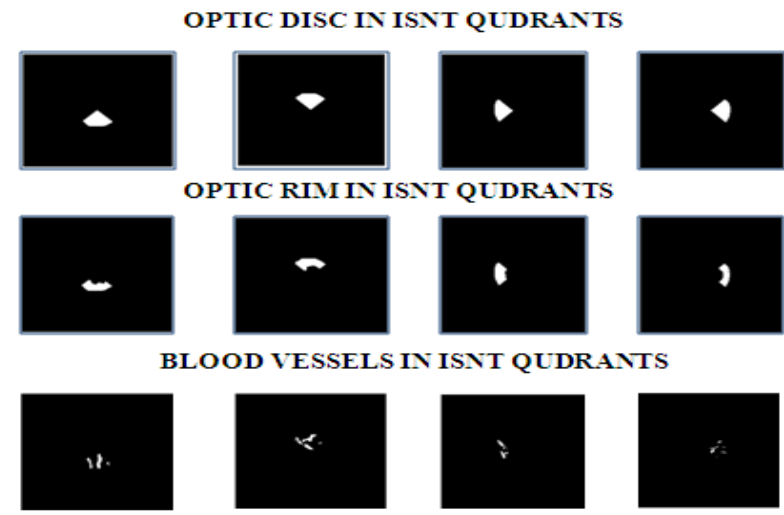

Fig 9: Segmented optic disc optic rim and blood vessels in ISNT quadrants

\section{CONCLUSIONS}

In this paper, a novel approach for the mask image generation for segmenting retinal fundus image features into ISNT quadrants has been presented. The proposed array centroid method have four steps, centroid calculation, array initialization, mask image generation and mask-feature image multiplication. The centroid of optic disc is calculated and then an array of size same as that of the image is initialized with all its elements zero. Then the masks are generated for all the four quadrants using the array characteristics and centroid value. The generated mask is multiplied with the feature image to obtain the quadrant segments. The proposed method accurately segments the optic disc, optic rim and blood vessels in disc region into ISNT quadrants.

The segmented features can be used for the ocular parameter calculation. The ocular parameters that can be calculated from the obtained features are Cup to Disc Ratio (CDR), Rim to Disc Ratio(RDR), Inferior, Superior Nasal, Temporal (ISNT) ratio etc.Further these parameters help in the diagnosis of eye disorders like glaucoma.

\section{ACKNOWLEDGEMENTS}

The authors are grateful to Dr. Rajesh Radhakrishnan. M. S Ophtalmic Surgeon and Glaucoma Consultant, Adithya Kiran Eye Care Centre Palakkad for providing the fundus image photographs and the guidance and support given for this work.

\section{REFERENCES}

[1]. C. B. Anusorn, W. Kongprawechnon, T. Kondo, S. Sintuwong and K. Tungpimolrut, "Image Processing Techniques for Glaucoma Detection Using the Cup to Disc Ratio," Thammasat International Journal of Science and Technology. vol.18, no.1, Jan-March 2013.

[2]. S. Kavitha and K. Duraiswamy "An Efficient Decision Support System For Detection of Glaucoma in Fundus Images Using ANFIS", International Journal of Advances in Engineering \& Technology vol.2, Issue.1, pp.227- 240, Jan. 2012

[3]. K. Narasimhan, Dr. K. Vijayarekha, “An Efficient Automated System For Glaucoma Detection Detection Using Fundus Image," Journal of Theoretical and Applied Information Technology vol.33, no.1 Nov. 2011.

[4]. K. Narasimhan , K.Vijayarekha,K. A. Jogi Narayana,P. S SivaPrasad and V. SathishKumar, "Glaucoma Detection $\mathrm{m}$ From Fundus Image Using Opencv," Research Journ al al of Applied Sciences, Engineering andTechnology, pp. 5459-5463, Dec. 2012.

[5]. S.Kavitha, S.Karthikeyan, Dr.K.Duraiswamy, "Neuroretinal rim quantification in Fundus images to Detect Glaucoma," IJCSNS International Journal of Computer Science and Network Security., vol.10,no.6, June 2010 [6]. J. Acharya, S.Gadhiya, K. Raviya, "Segmentation Techniques for Image Analysis: A Review," International 
Journal of Computer Science and Management Research. vol.2, Issue 1, pp.1218- 1221, Jan. 2013

[7]. P. K. Suryawanshi "An Approach to Glaucoma Using Image Segmentation Techniques" International Journal of Engineering Sciences \& Research Technology, pp. 2388-2392, Sep 2013.

[8]. J. Kaur, Dr.H.P.Sinha, "An Efficient Blood Vessel Detection Algorithm For Retinal Images Using Local Entropy Thresholding," International Journal of Engineering Research \& Technology, vol.1, Issue.4, pp.1-6, June. 2012.

[9]. S. Morales, V. Naranjo, J. Angulo and M. Alcaniz, "Automatic Detection of Optic Disc Based on PCA and Mathematical Morphology." IEEE Transactions on Medical Imaging, vol.32, no.4, April 2013.

\section{BIOGRAPHIES}

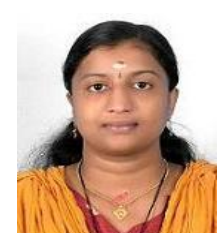

Darsana $\mathbf{S}$ received her $B$ Tech degree in ECE (first class with honours) from Nehru College of Engineering and Research Centre, Pampady, Thrissur, Kerala under University of Calicut in 2012. Presently she is doing MTech in Applied Electronics and Communication Systems in Nehru College of Engineering and Research Centre, Pampady, Thrissur, Kerala.

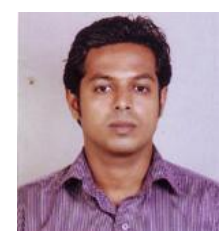

Rahul M Nair received his BTech degree in ECE from Ilahia College of Engineering and Technology, Muvattupuzha, Kerala under MG University in 2009 and MTech degree in VLSI and Embedded Systems from VJCET, Vazhakulam, Kerala under MG University in 2011. Presently he is working as Assistant Professor in Nehru College of Engineering and Research Centre Pampady Thrissur, Kerala. 